\title{
New Wolf-Rayet galaxies - IMF in starbursts
}

\author{
JieHao Huang, Q.S. Gu, and L. Ji \\ Astronomy Department, Nanjing University, Nanjing, 210093, China, \\ United Lab for Optical Astronomy, The Chinese Academy of Sciences, \\ 100080, China

\section{W. Zheng \\ Department of Physics and Astronomy, The Johns Hopkins University, Baltimore, MD 21218-2686}

\begin{abstract}
We report the discovery of two new Wolf-Rayet galaxies: Mrk 1039, and F8208+2816. Two broad WR bumps at $5808 \AA$ and $4650 \AA$ indicate the presence of WCE and WNL star populations in these two sources. Comparison of the observed EW(HeII $\lambda 4686)$ and EW(CIV $\lambda 5808)$ with recent models of WR populations in young starbursts provide an indication that the stellar initial mass function in some WR galaxies might not be Salpeter-like.
\end{abstract}

The stellar initial mass function is the fundamental quantity to study star formation processes and to understand the evolution of massive stars. Recent studies of the IMF in the Galaxy or in LMC and in starburts found a Salpeter-like IMF (Leitherer 1998; Scalo 1998), with slope $\alpha \approx 2.35$ in the form of IMF: $d N / d M \propto M^{-\alpha}$.

An interesting investigation conducted recently (Meyer 1996) found increasing high (1-10 $\left.\mathrm{M}_{\odot}\right) /$ low $\left(0.1-1 \mathrm{M}_{\odot}\right)$ mass ratio with increasing stellar density in six young clusters, implying a flat intermediate-mass IMF. This trend, however, is not confirmed in other three young regions, suggesting IMF variations above $1 \mathrm{M}_{\odot}$, as Larson (1998) or Scalo (1998) claimed. A possibility of locally top-heavy IMF in starbursts has been discussed in Larson's review, which is supported by observational evidence obtained recently in super-star clusters presented in this conference (Figer, these Proceedings).

The study of Wolf-Rayet galaxies could constrain the IMF slope to the mass range of $50-100 \mathrm{M}_{\odot}$. Previous studies show that the majority of observations of WR galaxies can be explained with models based on a canonical Salpeter IMF (Schaerer 1996; Mas-Hesse \& Kunth, private communication).

Among our newly discovered WR galaxies, Mrk 1039 and IRAS 08208+2816, might be worth studying in detail (Huang et al. 1999). Preliminary analyses show that the observed equivalent width (EW) of HeIr $\lambda 4686$ and CrV $\lambda 5808$ in these new sources are somewhat fitted by, or severaly discrepant with, model predictions based on a flat IMF $(\alpha=1.0)$ illustrated by the thick dashed line in Fig. 1.

Though the comparisons we made seem to indicate that the high-mass IMF in these new WR galaxies might not be Salpeter-like, a very flat IMF, $\alpha=1.0$, might be involved. However, the large uncertainties of about $40-45 \%$ in the measured equivalent widths make the conclusion vague! Obviously, more decisive 


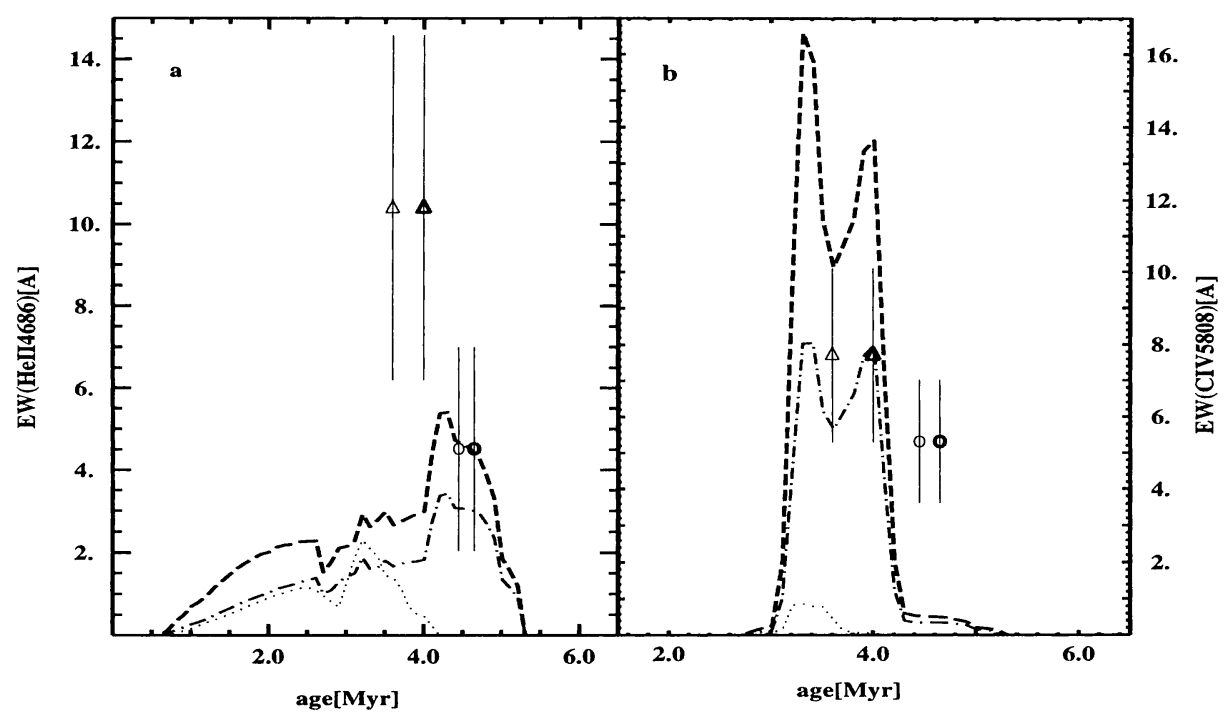

Figure 1. Comparisons between the predicted and observed quantities for $Z=0.004$ (dotted-dashed), 0.001 (thin dotted) based on Salpeter IMF, and $Z=0.004$ (thick dashed) based on a very flat IMF $(\alpha=1.0)$. (a) $E W$ of stellar HeII $\lambda 4686$; and (b) $E W$ of CIV $\lambda 5808$. Observed data are denoted by thick open symbols: triangle for Mrk 1039; and circle for F8208+2816. Thin open symbols are for related observed data corrected for mismatch of slit width with the nebular emission regions. Model predictions are taken from Schaerer \& Vacca (1998)

conclusion could not be drawn until obtaining higher resolution data with higher $\mathrm{S} / \mathrm{N}$ ratio, which are expected to come off in the near future.

\section{References}

Huang, J.H., Gu, Q.S., Ji, L., Li, W.D., Wei, J.Y., Zheng, W. 1999, ApJ 513, 215

Larson, R. 1998, MNRAS 301, 569

Leitherer, C. 1998, in: G. Gilmore \& D. Howell (eds.), The Stellar Initial Mass Function, ASP-CS 142, 61

Meyer, M.R. 1996, Ph.D. dissertation, U. of Massachusetts (see 1996, PASP 108, 380)

Scalo, J. 1998, in: G. Gilmore \& D. Howell (eds.), The Stellar Initial Mass Function, ASP-CS 142, 201

Schaerer, D. 1996, ApJ 467, L17

Schaerer, D., Vacca, W. 1998, ApJ 497, 618 